This document is the accepted manuscript version of the following article:

Jiang, S., Hailesilassie, B. W., Hean, S., \& Part1, M. N. (2020). Modelling structural response of flexible plug expansion joints under thermal movements. Road Materials and Pavement Design, 21(4), 1027-1044. https://doi.org/10.1080/14680629.2018.1534695

\title{
Modelling Structural Response of Flexible Plug Expansion Joints under Thermal Movements
}

\author{
Shunji Jiang ${ }^{1 *}$, Biruk W. Hailesilassie ${ }^{2}$, Sivotha Hean ${ }^{3}$, Manfred N. Partl ${ }^{4}$ \\ ${ }^{1}$ Structural Engineering, School of Civil Engineering, Georgia Institute of Technology, Atlanta, Georgia, United States, shun- \\ jijiang90@hotmail.com \\ ${ }^{2}$ Infrastructure Engineering, Division of Highway and Railway Engineering, School of Architecture and the Built Environment, \\ Royal Institute of Technology (KTH), SE-100 44 Stock-holm, Sweden, biruk.hailesilassie@abe.kth.se \\ ${ }^{3}$ Road Engineering/Sealing Components, EMPA, Swiss Federal Laboratories for Material Science and Technology, CH-8600 \\ Duebendorf, Ueberlandstrasse 129, Switzerland, Sivotha.Hean@empa.ch \\ ${ }^{4}$ KTH Stockholm, Division of Building Materials, Director Road Engineering/Sealing Components, EMPA, Swiss Federal \\ Laboratories for Material Science and Technology, CH-8600 Duebendorf, Ueberlandstrasse 129, Switzerland, \\ manfred.Partl@empa.ch
}

\section{Abstracts:}

This paper focuses on experiments and finite element modeling of flexible plug expansion joints (Asphaltic Plug Joints, APJ) subject to thermally induced horizontal movements. Five geometric and structural key parameters that influence (APJs) responses under thermal movements are studied: (1) joint length; (2) joint thickness; (3) joint width; (4) anti-bonding mat; (5) movement-aid spring. The viscoelastic computational finite element models are based on properties determined with a special cyclic coaxial shear test (CAST) and validated by an integrated approach incorporating cold temperature repeated movement capacity tests with a special Joint Movement Simulator (JMS) and a 3-Dimensional Digital Image Correlation system (3D DIC). It was found that the increase of joint width significantly reduces the stress at the interface between the mastic asphalt and APJ. The results also showed, that thin joints generate lower stress levels in APJ under thermal condition. Moreover, peak stresses in APJ appeared controlled mainly by the total size of the debonded region and the horizontal movement applied. The main findings are considered valuable for superior structural design, geometry selection and construction guidelines for APJ.

Keywords: Expansion Joint; Bridge, Pavement, Finite Element; Thermal movement; Debonding; Cracking. 


\section{Introduction}

Asphaltic Plug Joints (APJ) typically bridge the transversal gap between concrete bridge decks or between the bridge structure and natural ground, thus providing a smooth transition between the adjacent parts of the pavement, as typically shown in Figure1 according to the guidelines of Swiss Federal Road Office (ASTRA Guideline 2005). The primary serviceability requirements of an APJ system are to absorb bridge movements due to temperature variations and resist deformations due to dynamic traffic loads. APJs are also expected to reduce traffic noise, provide driving comfort and protect the bridge structure from water, de-icing substances and other corrosive liquids.

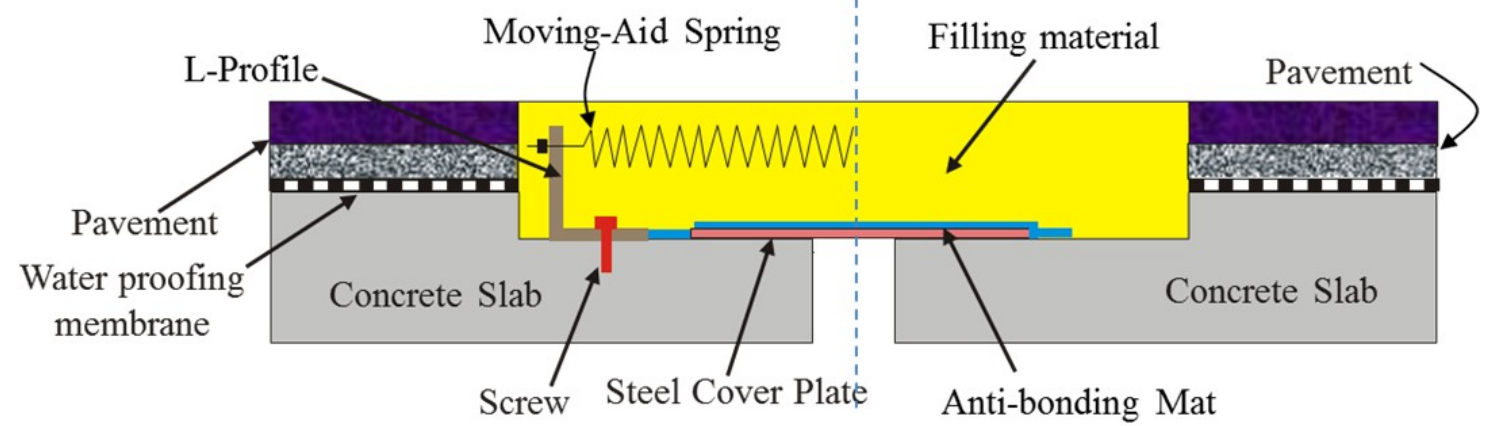

Figure 1 Schema of an APJ system for large movement (with movement-aid spring - left side) and small movement (without movement aid spring- right side).

Typical APJs consist of a trench filled with flexible joint filling material (mixture of aggregates and polymer bi-tumen binder) and a steel plate covering the gap between adjacent concrete bridge decks. Mechanical movement aids, such as mechanical springs, may be used for allowing absorption of large horizontal movements without significant premature failure (Partl, M. N. and Hean, S., 2011). Based on the Swiss ASTRA Guideline (2005), APJs for small movements below 30mm are typically 500mm wide and 100...130mm thick. APJs for large movements up to $100 \mathrm{~mm}$ may have a width between $500 \ldots 1000 \mathrm{~mm}$ and a thickness between $80 \ldots 140 \mathrm{~mm}$ but require mechanical movement aids in order to provide a homogeneous distribution of the joint movement along the joint width. The gap of the bridge joint is covered by a thin metal plate which is typically $150 \mathrm{~mm}$ wide for a $30 \mathrm{~mm}$ joint gap. It provides sliding ability and prevents the filling material to be squeezed into the gap by traffic loads. The springs of the movement aid are attached to the centroid of two L-profiles, which are mechanically anchored to the concrete bridge decks on both sides of the joint. Laboratory tests and field trials at EMPA confirmed that the embedding of the springs in the joint filling material is positive for a homogeneous strain distribution along thermal movements of joints (Partl, M. N. and Hean S, 2011). The friction between joint filling material and steel cover plate should be reduced to a minimum to avoid stress concentrations (Partl, M. N. and Hean, S, 2011). Hence, a thin layer of fabric acts as anti-bonding mat and covers the steel plate and the adjacent part of the joint/concrete interface.

As required by ASTRA Guideline ( 2005), APJs for small movements must be functional within $-20 \ldots+45^{\circ} \mathrm{C}$ for cyclic joint openings and closings between $20 \ldots-10 \mathrm{~mm}$. In addition, the maximum acceptable slope in any direction is $6 \%$ and the horizontal angle between the bridge axis and the longitudinal axis of APJs should not exceed $65^{\circ}$. With respect to bridge bearing replacements, APJs must also be able to endure at least $5 \mathrm{~mm}$ vertical gap movement. In 
case of APJs for large movements, the slope can be up to $10 \%$ and the horizontal angle up to $45^{\circ}$. Widespread popularity of APJs is challenged by frequently observed premature failures which are complex and relatively unpredictable. Failures may occur by combining different types of distress or by accumulating one particular severe distress. The APJ system fails when water and other contaminants can infiltrate to the substructure or when excessive deformation appears leading to a loss of driving comfort.

The three main failure modes with APJ systems are: debonding, cracking and rutting. Debonding refers to loss of adhesion at the vertical interface between APJ and the pavement. Two mechanisms proposed by Partl M. N et al., (2003) explain the causes of debonding. First, the impervious joint filling material will function as a water barrier and collect water at the vertical joint/pavement interface. During winter, the water may freeze and expand, thus causing debonding unless the collected water drains out properly. Second, the use of primers on the vertical APJ contact surface of wearing course may weaken the bonding strength at the interface between the pavement and APJ because the primer solvent may not fully evaporate or be absorbed by the wearing course. Cracking was frequently observed at low temperatures when the joint mixture became brittle and stress from traffic loads, joint movements and material fatigue exceeded the material capacity. Rutting and permanent deformation in the wheel path normally appears during summer. "Bumps" may occur when the joint material becomes soft at high temperature and is squeezed out by compression from thermal closing of the APJ, breaking and accelerating maneuvers.

\section{Objective}

APJ systems with movement aids are a promising and innovative solution against traffic noise in sensitive urban areas and in cases where large bridge movements must be absorbed. However, in order to understand the performance of APJ in practice, careful considerations of the interaction between movement aids and surrounding joint filling material are needed. Finite element modeling appears a useful inexpensive tool to establish movement capacity curves for various geometrical and structural configurations. It can also help in connecting test results with stress, strain, and temperature fields in the joints.

The objective of this study is to investigate the effects of five geometric and structural parameters on the behavior of APJ systems subject to thermally induced horizontal movements by focusing on experiments and finite element models with linear viscoelastic material properties. The five main variables in this parametric study are joint length, joint thickness, joint width, anti-bonding mat and movement aid spring. In order to determine the viscoelastic properties of the asphaltic joint filling material, the coaxial shear test (CAST) was conducted (Sokolov, K. et al., 2005). Experimental data was used for developing a constitutive model. The proposed finite element model was validated in an integrated approach incorporating cold temperature repeated movement capacity tests with a special Joint Movement Simulator (JMS) and a 3-Dimensional Digital Image Correlation system (3D DIC), demonstrating that this methodology can be used for future studies on similar joints

\section{Materials and Experimental Methods}

Joint filling materials typically consist of a mixture of gap-graded aggregates and polymer modified bituminous binder of a Styrene-Butadiene-Styrene (SBS) type. The use of polymer modified bitumen reduces the frequency of 
maintenance at particular locations of APJs and provides longer service life. As in a study by (Hean, S. and Partl, M. $\mathrm{N}, 2005$ ), a bitumen-rich mixture with binder content about $11 \ldots 25 \%$ by weight was used as joint filling material. For the adjacent pavement, dense mastic asphalt was used (Partl, M. N et al., 2002). Test procedures of binder, aggregates and APJ fillings are well defined in the Swiss Federal Road Office, ASTRA, guidelines (ASTRA Guideline 2005) and ETAG nº 032 (European Organisation for Technical Approvals, 2013).

\subsection{Co-Axial Shear Test (CAST)}

The Co-Axial Shear Test (CAST) has been designed by EMPA to determine mechanical properties and the complex stress-strain response of bituminous pavement layers under 3D loading conditions (Sokolov, K. et al., 2005). A cylindrical specimen with an outer diameter of $160 \mathrm{~mm}$ and a central hole with a diameter of $60 \mathrm{~mm}$ is glued between an external steel ring and a central steel core, as shown in Figure 2. Lateral deformations in the radial direction are constrained by the outer steel ring, which reflects the in-service boundary condition of a corresponding asphalt cylinder in a pavement. Sinusoidal loads are applied in the axial direction with a steel rod that is connected to the central steel core. Testing is performed in a temperature controlled climate chamber using a servo hydraulic testing machine.

Specimens consisted of a typical bituminous joint filling material and were conditioned in the climate chamber for at least 6 hours. Experiments were conducted at 8 frequencies in the range or $0.125 \ldots 16 \mathrm{~Hz}$ and at temperatures between $10 \ldots 20^{\circ} \mathrm{C}$. The dynamic modulus measurements will be discussed in Section 5.1 .

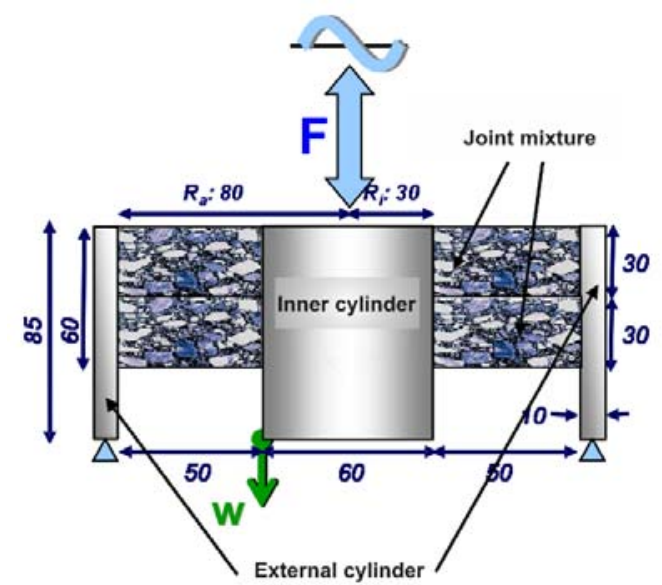

Figure 2 Schema of Co-Axial Shear Test (CAST) setup. 


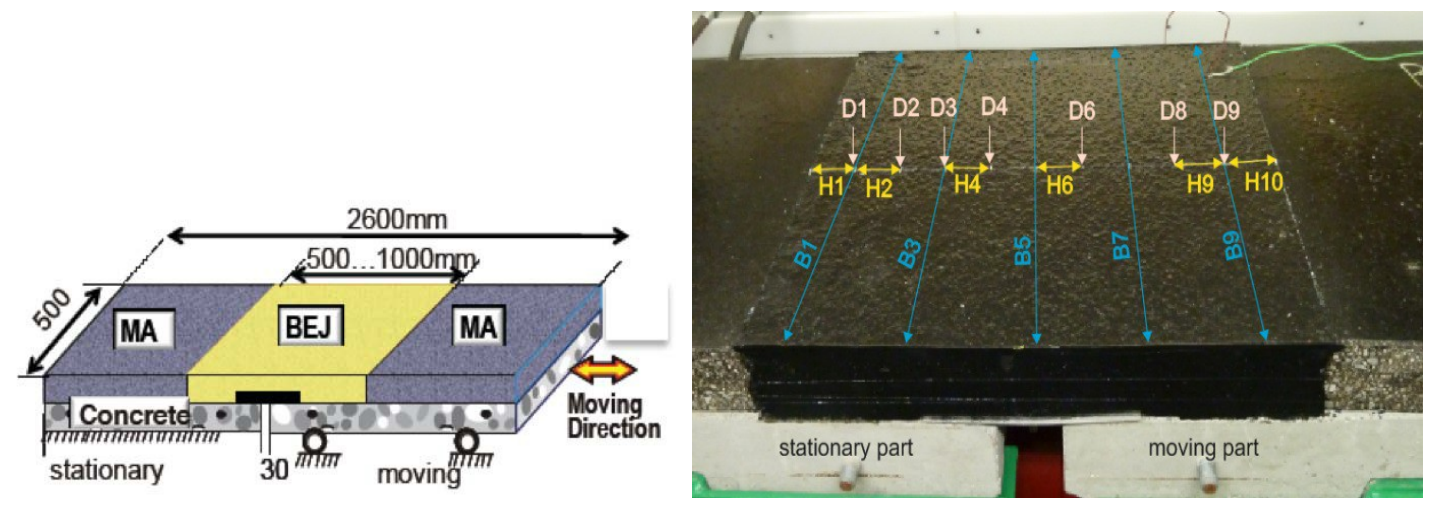

Figure 3 Schema of the JMS (left) and test setup with deformation markers on an APJ (right).

The special Joint Movement Simulator (JMS) shown in Figure 3 has been particularly developed for full-scale testing of APJs with respect to cold temperature repeated movement capacity. It consists of a stationary and sliding part on rails, which is horizontally moved by a hydraulic cylinder at constant displacement rate of $10 \mathrm{~mm} / \mathrm{h}$. Full-scale APJ specimens are conditioned and tested in a climatic chamber and equipped with embedded thermocouples for monitoring the specimen temperature during the test. The horizontal strain during movement is manually measured by determining the relative position of the deformation markers. Both force and specimen temperatures are continuously recorded with a sampling rate of 1/10s (Partl, M. N, et al., 2002).

The APJ full scale specimens were $500 \mathrm{~mm}$ wide and $75 \mathrm{~mm}$ thick. The anti-bonding mat exceeded the steel cover plate by $35 \mathrm{~mm}$ on each side. Cyclic tensile movements of $20 \mathrm{~mm}$, corresponding to $65 \%$ of the maximum admissible annual movement according to ASTRA Guideline (2005), were applied 20 times at $-20^{\circ} \mathrm{C}$, representing the coldest winter temperature in many areas of Switzerland. This JMS cold temperature repeated movement capacity tests can be considered as severe torture test since it is performed at extreme in-service temperatures assuming that one full possible annual tension cycle takes place at the lowest declared design temperature of APJ and at a fast joint movement rate of $10 \mathrm{~mm} / \mathrm{h}$ (Partl, M. N. and Hean, S, 2011).

\subsection{D Digital Image Correlation}

Digital Image Correlation (DIC) (Chu, T. et al., 1985) can provide a complete map of the displacement and surface strain fields and their evolution by successively comparing the position of randomly distributed speckles on the joint surface before and after deformation (Bing, P. et al., 2009). Measurements were performed with the 3D DIC system at EMPA that comprises two high-resolution cameras. Initial test calibration of the 3D DIC system was carefully conducted in a climatic chamber after having reached the desired test temperatures. A series of deformation images were captured at 1-minute intervals during the JMS cold temperature repeated movement capacity tests explained in Section 3.2. In order to smooth short-term fluctuations and highlight long-term trends 5-point moving average filter was applied. 


\section{Theoretical Approach}

\subsection{Material Constitutive Model}

The highly viscoelastic nature of the binder-rich joint filling material is the key for meeting the functional requirements of an APJ system while satisfying its serviceability requirements (Bramel, B. K. et al., 1999). For accurate prediction and detailed understanding the performance of APJs, finite element modeling with appropriate mathematical representation of the viscoelastic material properties was applied.

The complex shear modulus is determined and approximated by a five-parameter exponential Prony series given in Equation (1):

$$
G(t)=\sum_{k=1}^{K} G_{k} e^{-t / \tau_{k}}
$$

In Equation (1), $G(t)$ denotes the calculated shear modulus at time $t$; $\tau_{k}$ stands for the relaxation time; $G_{k}$ are the Prony coefficients with $k$ indicating the number of elements of the generalized Maxwell model.

The material is assumed thermo-rheologically simple, homogeneous and isotropic. Hence, the analytical expression (1) can be converted from time to frequency domain using Fourier transforms presented in Equation (2):

$$
G^{\prime}(\omega)=G_{0}\left(1-\sum_{k=1}^{K} g_{k}\right)+G_{0} \sum_{k=1}^{K} \frac{g_{k} \tau_{k}^{2} \omega^{2}}{1+\tau_{k}^{2} \omega^{2}}, G^{\prime \prime}(\omega)=G_{0} \sum_{k=1}^{K} \frac{g_{k} \tau_{k} \omega}{1+\tau_{k}^{2} \omega^{2}}
$$

Where $G^{\prime}(\omega)$ is the storage shear modulus and $G^{\prime \prime}(\omega)$ is the loss shear modulus both in frequency domain; $G_{0}, g_{k}$ and $\tau_{k}$ are Prony series parameters.

In addition, all other materials used in the finite element modelling herein were considered homogeneous and linear. The steel cover plate was modelled as elastic material with a modulus of 206,000 MPa. The stiffness of the mastic asphalt pavement was taken as temperature-dependent with 3,000 MPa at $20{ }^{\circ} \mathrm{C}$ and 7,000 $\mathrm{MPa}$ at $0^{\circ} \mathrm{C}$. The Poisson's ratio of mastic asphalt was assumed to be a constant of 0.35 for modelling purpose only. The concrete bridge deck was assigned a Young's modulus of 21,000 MPa.

\subsection{Finite Element Modeling using ABAQUS}

The three-dimensional finite element modeling (FEM) was carried out with ABAQUS 6.12. Two APJ prototype systems designated APJ- $1^{\mathrm{a}}$ (without movement aid spring) for small joint movements and APJ-2 ${ }^{\mathrm{a}}$ (with movement aid spring) for large movements are discussed in the following parametric study. In addition, one thin APJ-1 ${ }^{\mathrm{c}}$ and one APJ-1 ${ }^{\mathrm{b}}$ without anti-bonding mat for small movements were considered as summarized in Table 1.

Table 1 Dimensions of FEM models

\begin{tabular}{|c|c|c|c|}
\hline Model ID & Width [mm] & Thickness [mm] & Length [m] \\
\hline APJ-1 $^{\mathrm{a}}$ & 500 & 100 & \multirow{2}{*}{0} \\
\cline { 1 - 2 } APJ-1 $^{\mathrm{b}}$ & 500 & 100 & \multirow{2}{*}{0.5} \\
\cline { 1 - 3 } APJ-1 $^{\mathrm{c}}$ & 500 & 75 & \\
\hline APJ-2 $^{\mathrm{a}}$ & 500 & 100 & \\
\hline
\end{tabular}


APJ-1 $1^{\text {a }}$ Prototype of APJ for small movement;

APJ-2 ${ }^{\text {a }}$ Prototype of APJ for large movement.

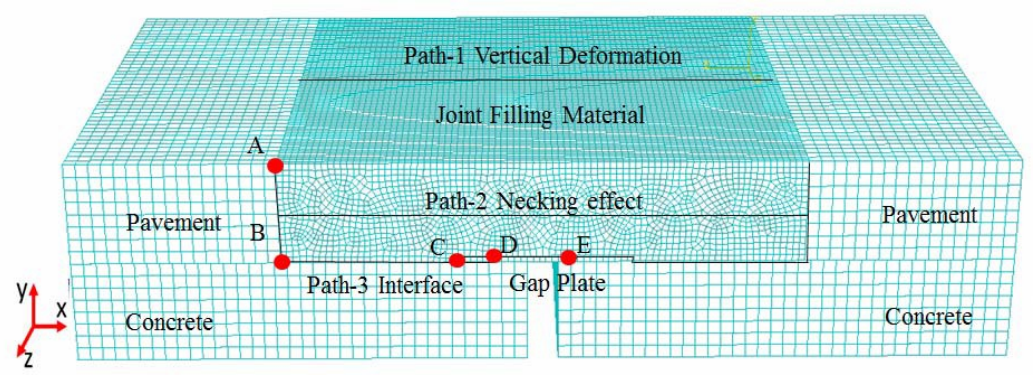

Figure 4. 3D finite element model APJ-1 ${ }^{\mathrm{a}}$; Locations of interest and results-probing paths

Eight-node isoparametric elements (Q8) with reduced integration, known as C3D8R in ABAQUS, were used to mesh the whole model according to Figure 5. In order to ensure meaningful results, the mesh was improved by refining the mesh at critical locations such that more elements appeared where field gradients were high and more resolution was required. The mesh was refined to ensure a maximum element size of $6 \mathrm{~mm}$ in the joint, where cracks and debonding were expected to initiate, albeit a larger element size of $12 \mathrm{~mm}$ was assigned to pavement and concrete. Quantities of interest within each element were averaged over the element domain. However, the stresses and strains were most accurate at the integration point that was located in the middle of the element. Hourglass control was activated to alleviate spurious zero energy modes. Generally higher order formulations such as 4 node quadratic elements are better than triangular elements. However, since the geometry in this particular case was complicated (spring and the APJ as shown in Figure 15), triangular meshing had to be used instead of quadratic elements. Mesh density analysis was done for the model before proceeding to the analysis. It was found that the mesh size was resulting in a stable output.

In order to facilitate comparisons between various configurations, three resulting-probing paths were created as indicated in Figure 4. Path-1 rests on the top surface of joint to evaluate vertical surface deformation and horizontal strain distribution; Path-2 locates on the side surface to examine the lateral contraction of side surfaces; Path-3 covers half of the APJ system interface due to geometry and loading condition symmetry, indicating stress distribution and stress concentration at the following critical locations: (A) joint-pavement contact location on the traffic exposed surface, (B) contact location of three materials (structural concrete, mastic asphalt of the pavement and joint filling material) at the bottom of the plug joint, (C) end of the anti-bonding mat, (D) edge of the steel cover plate, (E) central bottom location of the joint on top of the steel cover plate..

\subsubsection{Interaction between APJ components}

The contacts between concrete and asphalt mastic of the pavement, concrete and joint filling material, joint filling material and mastic of the pavement and, in case of joints with moving aids, between concrete and L-profile as well as joint filling material and L-profile are assigned with boundary condition of permanent adhesion at the joint-pavement interface. In other word, failure will occur inside the material and not at the interface. There is evidence in Bramel, B.K. et al. (1999) and ASTRA Guideline (2005) that this is a reasonable assumption. It should be noticed that the intent of our study was to simulate stress and strain fields for detecting critical locations where the risk of cracking and debonding in APJ was high. Debonding, crack initiation and propagation phenomena in the APJ associated with time and temperature dependencies including healing of bituminous material as well as material non-linearity, as discussed 
e.g. by Di Benedetto et al. (2001), were out of the scope of this study but should certainly be considered in future studies.

Debonding around the steel cover plate is an influential factor on APJ performance. The anti-bonding mat covers this steel plate and reaches into the joint-concrete interface at each side of the plate by a constant distance of $35 \mathrm{~mm}$ for all models in this paper. Everything below the anti-bonding mat is considered as debonded. When thermal movement is applied, the cover plate is assumed to slide freely under the anti-bonding mat. Even though the anti- bonding mat does have influence on the adhesion behavior, it has a negligible influence on the stiffness of the APJ system. Furthermore, following the construction process recommended by the design team, no mechanical fixation was assumed between steel plate and concrete deck. Therefore, both the top and bottom surface of steel cover plate are ideally modelled with frictionless contact.

3D spring geometry was created in CATIA V4 and imported into ABAQUS for assembly. Five springs in a row were embedded in the joint with a spacing of $100 \mathrm{~mm}$ and a distance of $50 \mathrm{~mm}$ to both lateral boundaries of the APJ. The springs with $29 \mathrm{~mm}$ outer diameter and $416 \mathrm{~mm}$ length had a wire diameter of $3.2 \mathrm{~mm}$ and were assumed linear elastic. The interaction between spring and joint filling material was modeled as solid-in-solid embedded constraint (Taylor R. L. et al., 1970). The joint part acted as host region and constrained the response of embedded region.

\subsubsection{Loading and boundary conditions}

In reality, the APJs are subjected to a combination of traffic load, seasonal thermal movements of the bridge deck as well as thermal movements from heat transfer during daily temperature fluctuations. However, the dominant APJ load case considered herein is thermal movement of the bridge, assuming that the thermal movement of the bridge is slow enough to be considered as quasi-static and the APJ remains in thermal equilibrium such that heat transfer due to daily temperature variations can be neglected. Prescribed displacements were applied to the APJ boundaries.

The vertical displacement was taken as constrained on the bottom surface of concrete. Horizontal movement with a velocity of $10 \mathrm{~mm} / \mathrm{h}$ was applied on the right surface of both concrete and mastic asphalt. All degrees of freedom were considered fixed on the left surface of the concrete and mastic asphalt. As indicated in the ASTRA guidelines, APJ systems for small movements should be able to absorb an annual movement of $20 \mathrm{~mm}$ opening and $-10 \mathrm{~mm}$ closing within a temperature range from $-20^{\circ} \mathrm{C}$ in winter to $+45^{\circ} \mathrm{C}$ in summer. Therefore, two cases of thermal movements were studied in this paper: firstly, a $20 \mathrm{~mm}$ opening with a predefined temperature field at $-20^{\circ} \mathrm{C}$; secondly, a $-15 \mathrm{~mm}$ closing with a $+45^{\circ} \mathrm{C}$ predefined temperature field. 


\section{Results and Discussions}

\subsection{Material Characterization}

As stated in Section 3.1, from the applied load and measured deformation with the Co-Axial Shear Test, the dynamic modulus and phase angle data of the APJ material were determined as input for constructing the master curve in Figure 5 for a reference temperature of $20^{\circ} \mathrm{C}$.

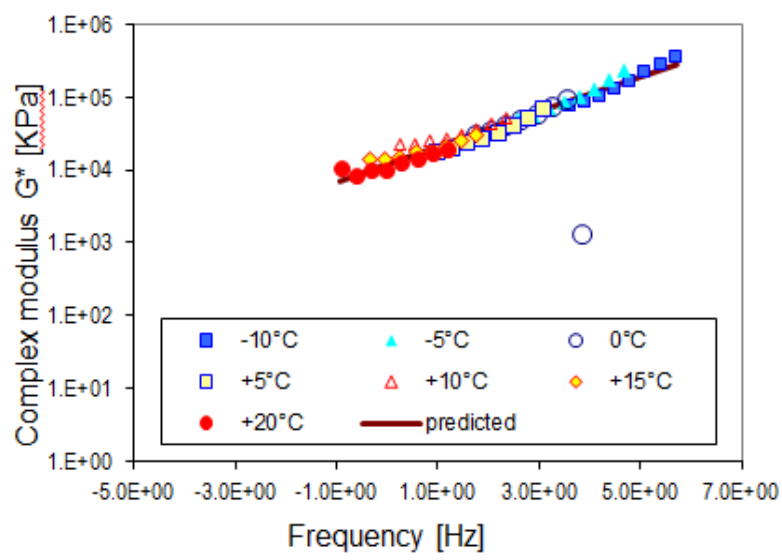

Figure 5 Co-axial Shear Test results: master curve for a reference temperature of $20^{\circ} \mathrm{C}$

In order to obtain the Prony series parameters for facilitating finite element modeling, an optimization algorithm in MATLAB was used for minimizing the residual of the difference between the CAST experimental data and the Prony series. Pre-smoothing was conducted for obtaining better result, by first fitting the master curve to a power-law function. The Prony series parameters are presented in Table 2. They provide a good fit for the entire master curve constructed from CAST experimental data, see Figure 6.

Table 2. Prony series model parameters (5 terms), at $\mathrm{T}_{\text {ref }}=20^{\circ} \mathrm{C}$

\begin{tabular}{cccc}
\hline$i$ & $\begin{array}{c}G_{0} \\
{[\mathrm{MPa}]}\end{array}$ & $g_{i}$ & $\tau_{i}$ \\
\hline 1 & & 0.7298 & 0.0004 \\
2 & & 0.1338 & 0.0044 \\
3 & 435.0859 & 0.0593 & 0.0114 \\
4 & & 0.0462 & 0.7853 \\
5 & & 0.0167 & 25.7939 \\
\hline
\end{tabular}




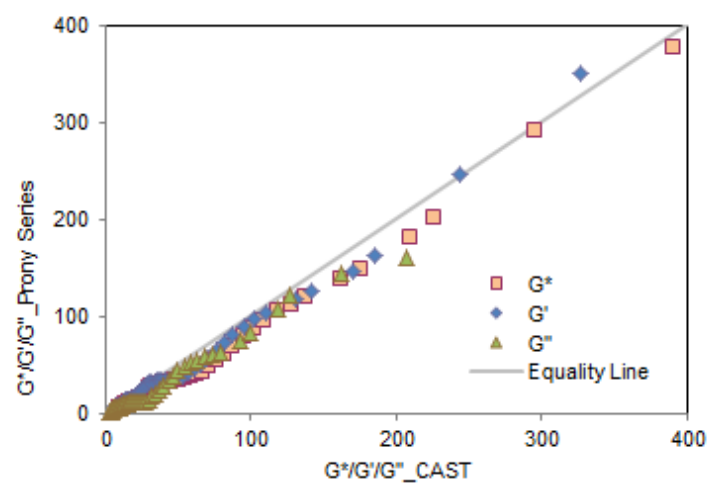

Figure 6 Prony series data curve fitting

\subsection{Finite Element Model of APJ}

Finite element simulations using model $\mathrm{APJ}-^{\mathrm{C}}$ are discussed focusing on results for the selected paths indicated in Figure 4: (1) Vertical deformation along y-axis; (2) Lateral contraction (necking deformation) along z-axis; (3) horizontal strain along X-axis; (4) von Mises stress along the APJs interfaces.

According to Bramel B.K. et al. (1999) and Partl, M. N. and Hean, S. (2011), there are two typical crack initiation and propagation paths leading to functional failure of APJ: (1) along the interface path ABCDE; (2) crack initiating from $\mathrm{C}$, the end of the anti-bonding mat or the edge of steel plate, and propagating vertically to the joint traffic exposed surface. Water penetration and aging will give rise to severe damage of APJ system, or even destroy bridge structural component beneath the APJ.

\subsubsection{Comparison with 3D DIC Deformations during JMS and Testing}

The displacement contours from the 3D DIC are compared with the finite element modeling, as demonstrated in Figure 7 and 8. Comparable displacement distribution pattern were observed, which reflects that actual deformations and stain distributions could be well predicted with FEM.

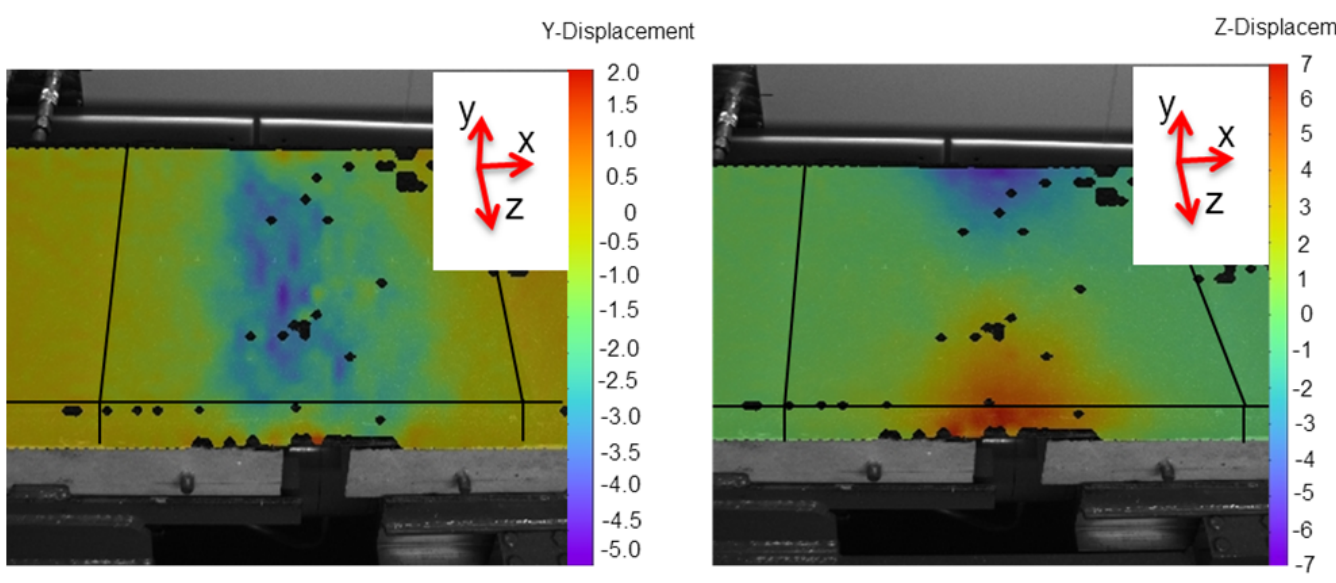

Figure 7 3D DIC: Vertical deformation along y-axis (left); Lateral contraction along z-axis (right): subjected to $+20 \mathrm{~mm}$ joint expansion in the JMS test at $-20^{\circ} \mathrm{C}$. 

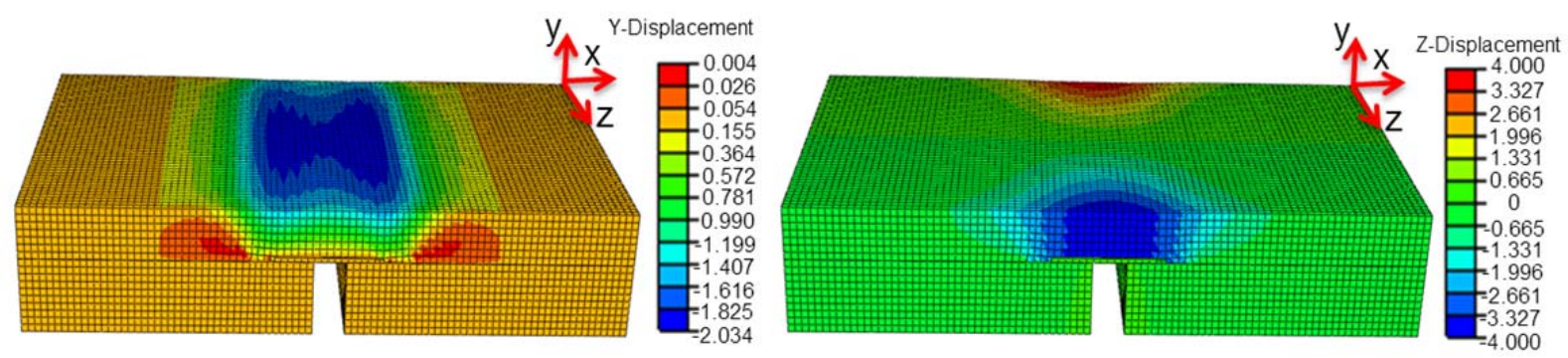

Figure 8 FEM: Vertical Deformation along y-axis (left); Lateral contraction along z- axis (right): subjected to $+20 \mathrm{~mm}$ joint expansion at $-20^{\circ} \mathrm{C}$.

Additional validation of the model was conducted by comparing model results along the joint width with manual measurements and 3D DIC data during a cold temperature repeated movement capacity test using JMS, as shown in Figure 9. While the maximum necking deformation shows a difference of 36\% between FEM and JMS measurements, the overall FEM simulated vertical and necking deformation is reasonably consistent with those from JMS manual and 3D DIC measurements, given the uncertainties regarding actual material parameters and precision of manual measurements. Furthermore, as shown in Figure 9c, horizontal strain concentrates on the middle part of the joint with an obvious strain peak of 0.1. Based on Harvey, J.A.F. and Cebon, D. (2003 ), tensile failure strains could be divided into three categories: ductile $(>1.0)$, transition, and brittle failure $(<0.1)$. Figure 9c indicates that the observed strains fall into the brittle failure region, which is not surprising for the JMS test condition with accelerated strain rate and testing temperature lower than the glass transition temperature of the binder. The results indicate that the calibrated APJ model captured the distribution patterns of deformations reasonably well and proved to be a proper tool for predicting APJs response for various geometric and structural configurations. However, there is still room for improvement and future research by introducing refined material properties (e.g. in terms of Poisson's ratio) in a more detailed and sophisticated FEM modelling framework. 


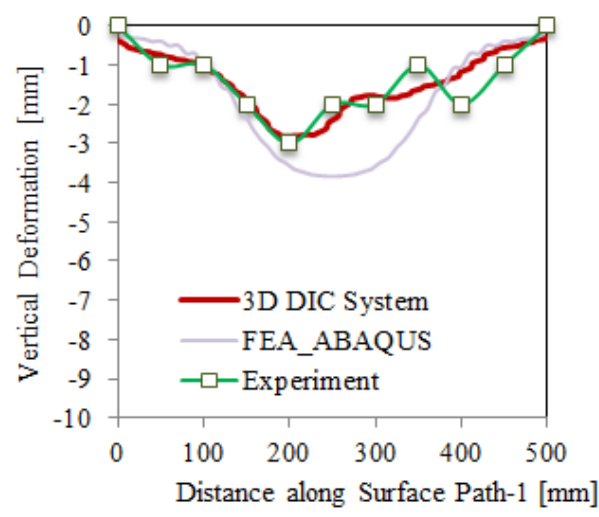

(a)

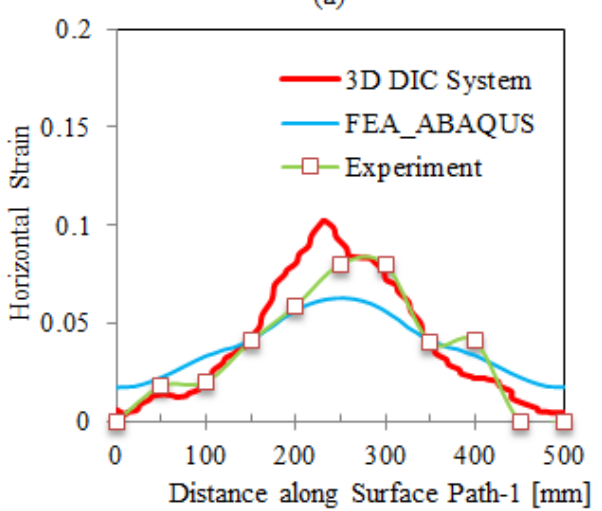

(c)

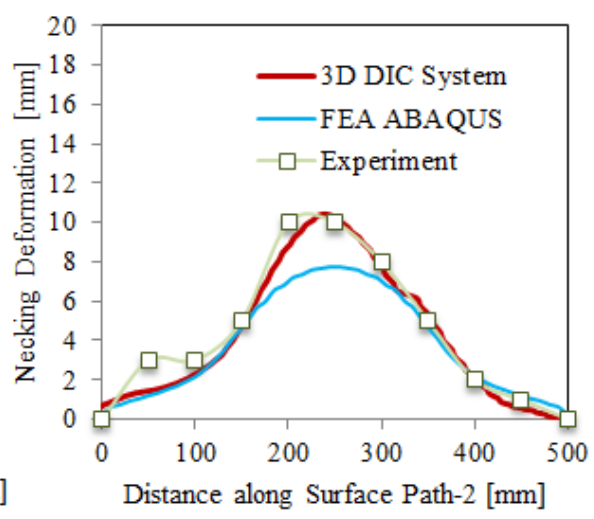

(b)

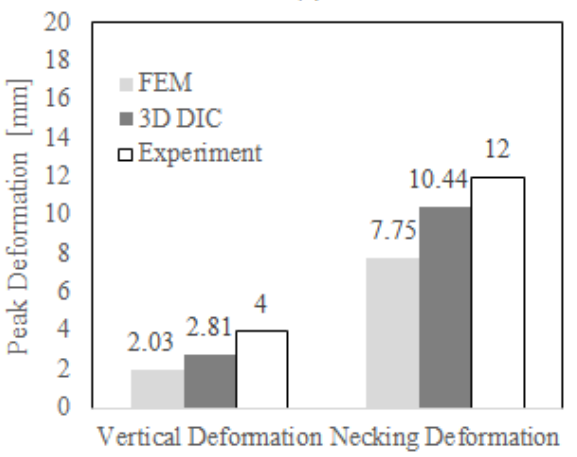

(d)

Figure 9 Comparison among FEM simulation, JMS manual and 3D DIC measurement: (a) Vertical deformation along joint width in y-axis at the center; (b) Necking deformation along z-axis at the edge; (c) Horizontal strain along x-axis; (d) Peak deformations: subjected to $+20 \mathrm{~mm}$ joint expansion at $-20^{\circ} \mathrm{C}$.

\subsection{Parametric Study}

\subsubsection{Joint Length}

Joint length is one main parameter to evaluate the size effect of the specimens tested in the laboratory. In the cold temperature repeated movement capacity test, the front and back side surfaces of the joint specimen have free boundaries and natural confinement provided by adjacent structures (natural ground, pavement shoulder, curb \& gutter etc.) is ignored. Generally, for practical testing reasons, it is assumed that specimens with a constant length of $500 \mathrm{~mm}$ are sufficiently representative for simulating the performance of in-service APJ systems, which are typically 8m wide for a two-lane street. Four comparable APJ models were discussed, which have the same configuration as prototype model (APJ-1 $1^{\mathrm{a}}$ ) in $\mathrm{x}-\mathrm{y}$ plane but with different joint length of $0.3 \mathrm{~m}, 0.5 \mathrm{~m}, 1 \mathrm{~m}$ and $2 \mathrm{~m}$ along the z-axis, see Table 1.

As indicated in Figure 10a and 10c, the vertical deformation along top surface path-1 and the horizontal strain along the interface path-3 change little with various joint lengths. However, obvious differences can be observed in the lateral necking deformation, as indicated in Figure 10b. There is nearly no change in necking when the joint length is larger than $1 \mathrm{~m}$. However, for shorter lengths, 38\% and 16\% differences in peak necking deformation are found in case of $0.3 \mathrm{~m}$ and $0.5 \mathrm{~m}$ length respectively. 


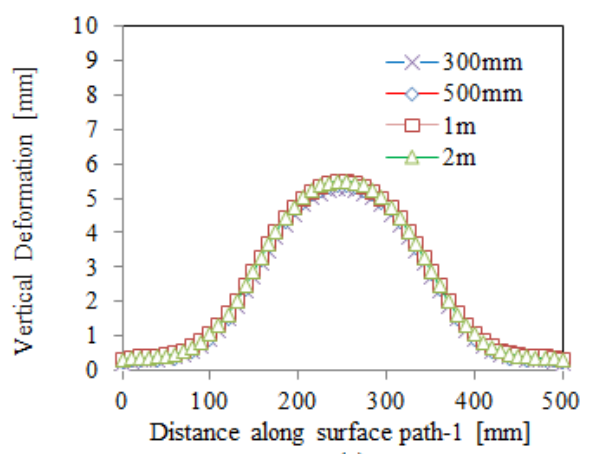

(a)

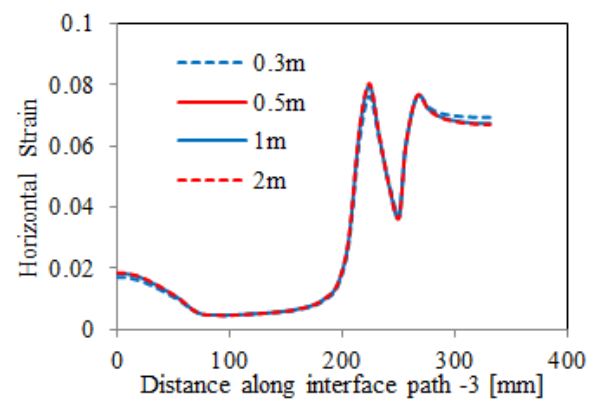

(c)

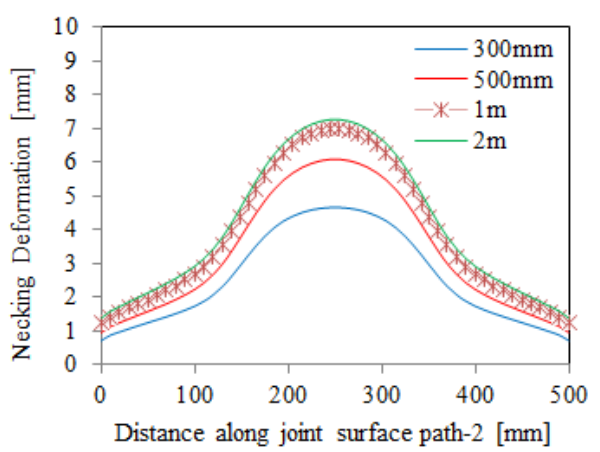

(b)

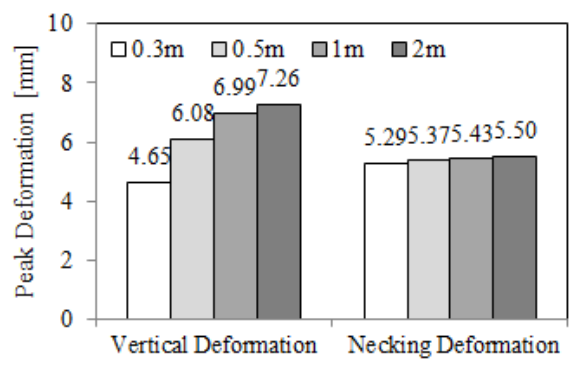

(d)

Figure 10 Variation of (a) vertical deformation along y-axis; (b) necking deformation along z-axis; (c) horizontal strain along $\mathrm{x}$ - axis; (d) peak deformations due to different joint length: subjected to $+20 \mathrm{~mm}$ joint expansion at $20^{\circ} \mathrm{C}$.

\subsubsection{Joint Width and Thickness}

Joint width and thickness are greatly influencing the pattern of joint failures. Eight models using APJ-1 as prototype were compared and used for constructing the response curve as a function of geometry. Joint width varied from $300 \mathrm{~mm}$ to $600 \mathrm{~mm}$ and was combined with joint thicknesses of $100 \mathrm{~mm}$ and $60 \mathrm{~mm}$.

From Figure 11 follows, that von Mises stresses at Point A and B decrease with wider and thinner APJ systems. However, stress at location $\mathrm{C}$ and $\mathrm{E}$ varies only little with joint width and thickness under thermal movements. This means that the localized stress concentration at the contact nonlinearity point is dominated by the total debonded length.

Thinner and wider joints are superior when only thermal movements are taken into account. With decreasing thickness and increasing width, stress at joint/pavement interface A-B drops dramatically and becomes significantly lower than at C. Hence, the crack is most likely initiated directly at the steel cover plate or at the end of the anti-bonding mat before propagating vertically to the surface. Thin joints are more vulnerable to stress concentration around CDE and more sensitive to the total debonded length.

Stress distribution for narrow joints may follow two possible failure patterns: (a) Bottom-up crack initiated from the end of anti-bonding mat or (b) debonding from steel cover plate because of peak stress at point C. However, it is also highly possible that narrow joints may fail at the joint-pavement interface, or further away from the edge of the gap plate, where the stress difference between $\mathrm{A}$ and $\mathrm{C}$ becomes less dramatically different. 

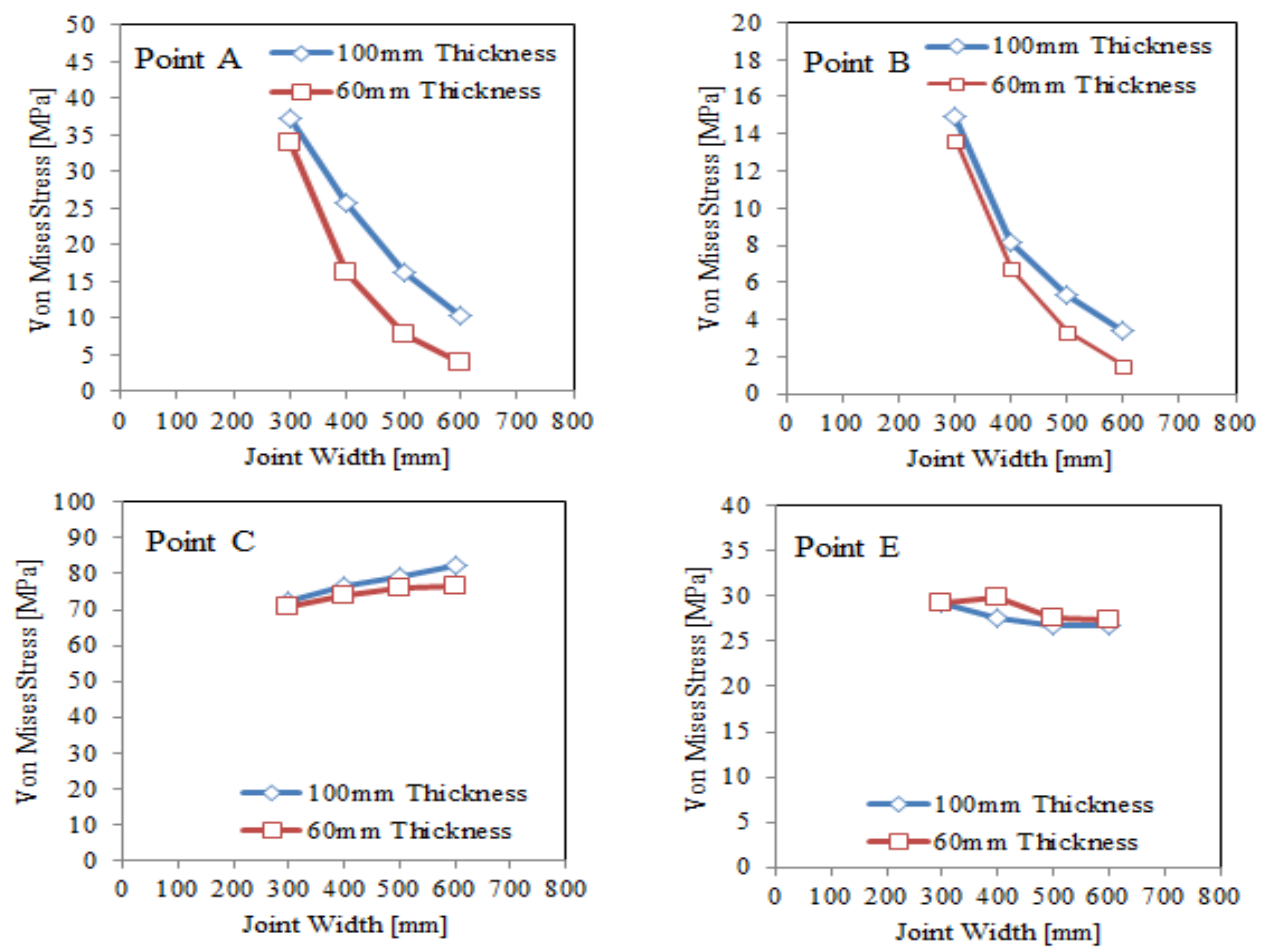

Figure 11 Variation of von Mises stress at critical locations A, B, C and E with variation of joint thickness and width: subjected to $+20 \mathrm{~mm}$ joint expansion at $-20^{\circ} \mathrm{C}$.

\subsubsection{Anti-bonding Mat}

The effect of an anti-bonding mat is investigated through two comparable models APJ- $1^{\mathrm{a}}$ and APJ- $1^{\mathrm{b}}$. All the finite element simulations are run at a constant strain rate of 5.56E-06/s. Remember that APJ-1 $1^{\mathrm{b}}$ intends to simulate an APJ system for small movements without any anti-bonding mat.
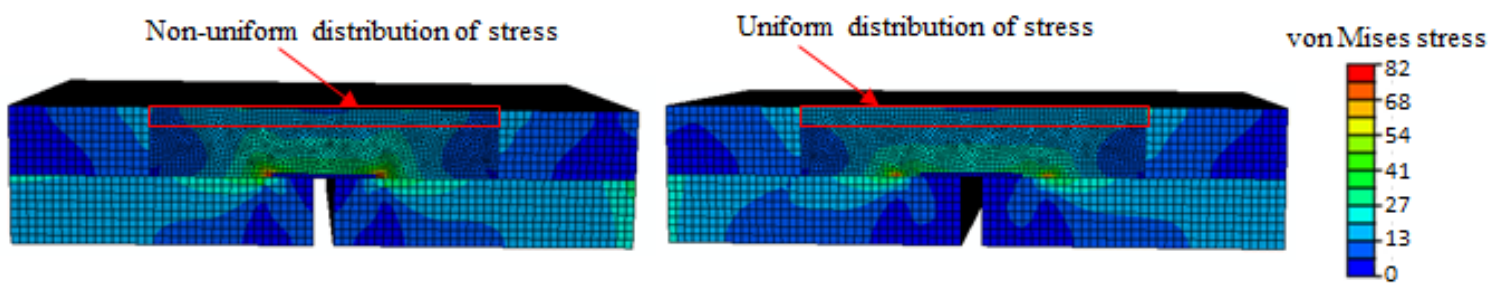

Figure 12. Von Mises stress contour of $A P J-1^{b}$ without anti-bonding mat (left) and APJ-1 $1^{\mathrm{a}}$ with anti-bonding mat (right).

Figure 12 shows a comparison of von Mises stress contour map between APJs with and without anti-bonding mat. In $\mathrm{APJ}-1^{\mathrm{b}}$, the most severe stress concentration occurs within the joint/steel cover plate interface. The high stress concentration level indicates that cracks will most likely initiate from the edge of the steel plate and propagate bottomup to the surface. Park, P. et al. (2010) stated that debonding at the edge of the steel cover plate is physically unavoidable when thermal movements occur. In reality, thermal movements make the steel cover plate and concrete deck move in opposite directions, and debonding between joint bottom surface and top of the steel plate 
automatically starts from the edge of the steel cover plate.

However, for APJ-1 $1^{\mathrm{a}}$ with anti-bonding mat, stress is distributed more uniformly in the whole joint material. This means that the potential of the viscoelastic joint to absorb movements is higher. It is clear that point $\mathrm{A}, \mathrm{B}, \mathrm{C}$ and $\mathrm{D}$ are stress concentration locations. The maximum stress location automatically moves from the edge of the steel cover plate (Point D in APJ-1 ${ }^{\mathrm{b}}$ ) to the end of the anti-bonding mat (Point C in APJ-1 $1^{\mathrm{a}}$ ).

According to Figure 13, the anti-bonding mat has no considerable effect at the interface between the mastic asphalt and the APJ. This is shown, for instance, at point A (top free corner intersection) and point B (bottom tri- material intersection), where the stress results are comparable. As depicted in Figure 13, the maximum stress along the path shifts from point $\mathrm{C}$ to $\mathrm{D}$ when removing the anti-bonding mat. However, the maximum stresses in both cases are comparable. Moreover, from Figure 12 follows that the von Mises stresses found close to the surface of the APJ are more uniformly distributed with than without anti-bonding mat.
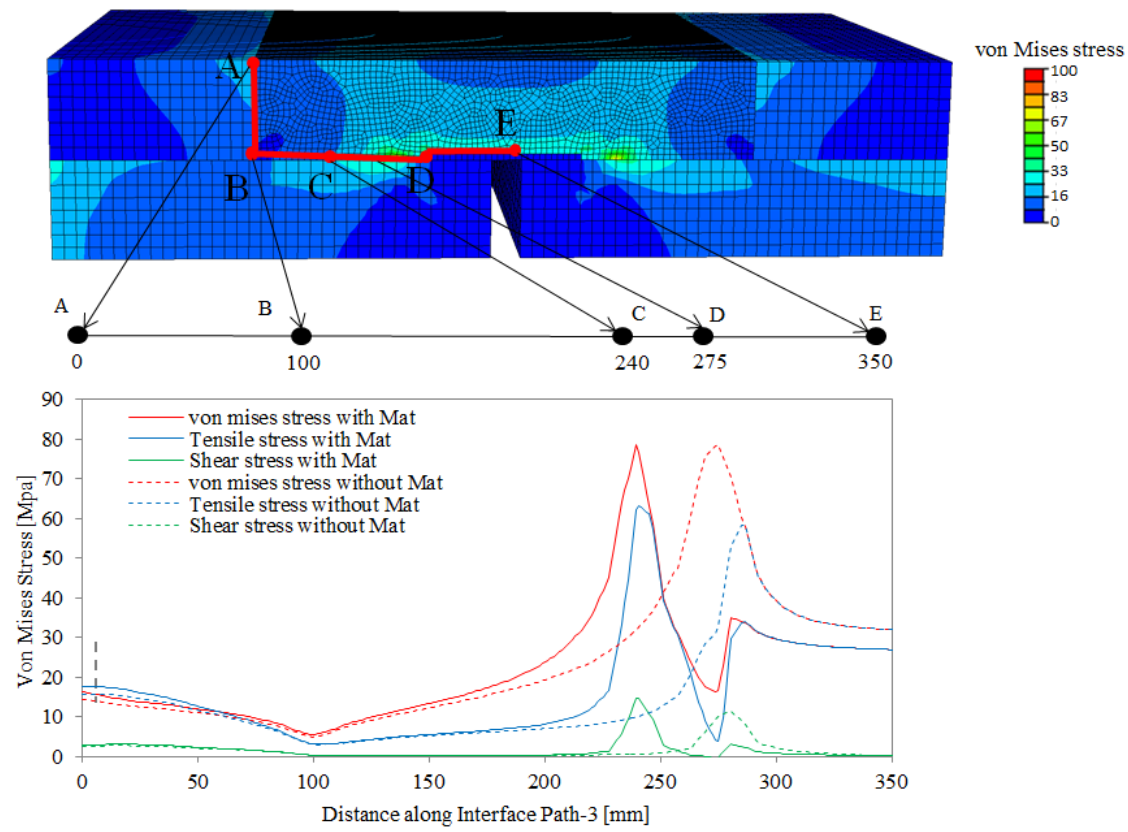

Figure 13. Variation of stress distribution along joint interface path with and without anti-bonding mat: Von Mises stress, tensile stress and shear stress subjected to $+20 \mathrm{~mm}$ joint expansion at $-20^{\circ} \mathrm{C}$. 


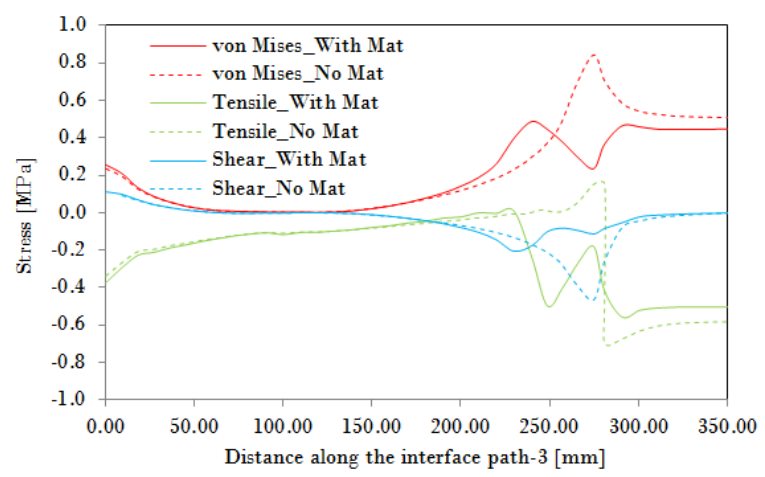

Figure 14. Variation of stress distribution along joint interface path with and without anti-bonding mat: Von Mises stress, tensile stress and shear stress subjected to $-15 \mathrm{~mm}$ joint contraction at $+45^{\circ} \mathrm{C}$.

In case of contraction, a typical thermal condition with $15 \mathrm{~mm}$ joint closing under $+45^{\circ} \mathrm{C}$ was simulated on both APJ1a and APJ-1b. Von Mises stresses along the interface path-3 are presented in Figure 14. Using a fabric mat reduces the stress magnitude along the whole joint interface path-3. Additionally, the stress within APJs under compressive movement is small as compared to tensile movement. Therefore, tensile thermal movements are the reason for the high stress levels in the joint. Under compression movements, strain related APJ failure criteria such as acceptable bump size have to be considered. With an anti-bonding mat, bending-like deformations appeared as a function of debonded length. Hence, excessive deformation can lead to APJ failure due to loss of flatness. Vertical deformation along $y$-axis was examined along surface path-1. The center of the joint bumps up vertically by $6.28 \mathrm{~mm}$ which is about $1 / 3$ of the allowable bump size of $19 \mathrm{~mm}$ proposed by (Bramel, B.K. et al. (1999) based on a survey conducted in United States.

\subsubsection{Movement-aid spring}

APJs containing movement-aids have advantages with respect to the adhesion stress situation at the interface between the joint filling material and the adjacent pavement (Partl, M. N. and Hean S, 2011). Figure 15 shows the von Mises contour of $\mathrm{APJ}-2^{\mathrm{a}}$ subjected to $20 \mathrm{~mm}$ joint opening at $-20^{\circ} \mathrm{C}$. It provides a rough idea on the function mechanism of a specific movement-aid system. The majority of the stress was transferred through the mechanical fixation with steel L-profiles and was taken over by the movement-aid springs. Therefore stress demand at vertical interface A-B is reduced and the whole system is less vulnerable to top-down cracking under low temperature conditions. The distance between the L-profile and the vertical interface A-B needs to be large enough to ensure proper filling with joint material. In addition, the height of L-profiles should be properly adapted to the thickness of the joint to prevent bottom-up cracking initiated from the top of the L-profiles. Further research is needed to provide better insight into the effect of different configurations of movement-aids and fixation conditions. 


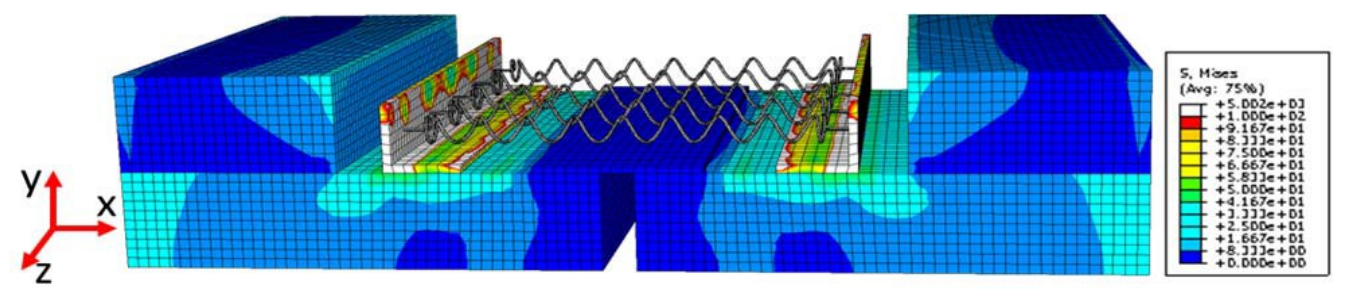

Figure 15 Model APJ-2: APJ model for large displacements with movement-aid spring.

In order to understand the benefits of movement-aid springs, APJ without and with movement-aids, i.e. APJ- $1^{\mathrm{a}}$ and APJ-2 ${ }^{\mathrm{a}}$, were compared. Von Mises, tensile and shear stresses were calculated along the interface path-3, as presented in Figure 16, leading to the following observations: (1) On the joint/pavement interface, the von Mises stresses at both the top and bottom points A and B drop 43\% and 56\% respectively. This is in accordance with the assumption stated earlier that the movement-aid springs decrease the stress demand at the joint/pavement interface. (2) $9 \%$ of the von Mises stresses were reduced at C, which marks the end of the anti-bonding mat. Hence, the movement-aid spring has an insignificant stress reduction effect on Point C. This makes sense because stress concentration at Point $\mathrm{C}$ is controlled by total debonding length. (3) However, at position D and E around the steel plate, the von Mises stresses increase 6 7\%. Again, this is considered acceptable because the von Mises stress contour of joint fillings in Figure 17 shows clearly local stress disturbance around the spring wire. Hence, the stress distribution in the joint filling material is non-uniform, resulting in local rise of stress and local stress concentration. (4) The tensile stress decreases significantly in the region between C-D when the movement-aid spring is used, which indicates the region of APJ filling material between the two L-Profiles mainly required to transfer the shear forces into the concrete. A long antibonding mat that covers the whole area between the edges of two L-shaped profiles is recommended for an APJ system for large movements since high stress levels are expected in the region between C-D.

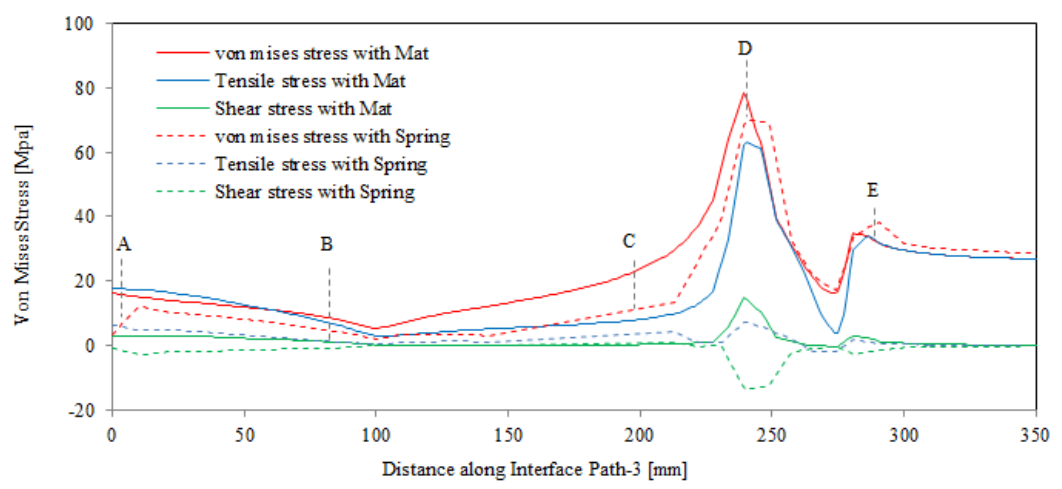

Figure 16. Variation of stress distribution along joint interface path-3 from APJ-1 ${ }^{\mathrm{a}}$ and APJ-2 ${ }^{\mathrm{a}}$ : Von Mises Stress, tensile stress and shear stress subjected to $+20 \mathrm{~mm}$ joint expansion at $-20^{\circ} \mathrm{C}$.

The local effects of the spring spirals were studied in more detail by creating 3 stress-probing paths at different heights within the joint fillings as shown in Figure 17. Path 4 and 6 follow the top and bottom surface of the joint filling. Path5 is right on top of the spring. Obviously, clear stress disturbances around the spring wire are present. Several other observations can be made: (1) at locations where the spring wire is embedded, a valley in the stress distribution of the 
joint filling occurs. (2) The overall distribution trends along these three paths shows that some windings in the middle of the spring carry more stress and strain than those near the two ends. (3) The bottom part of the joint is most dramatically affected by the embedded springs. Moreover, the stress waves in the joint become smoother with larger distance from the spring wire.

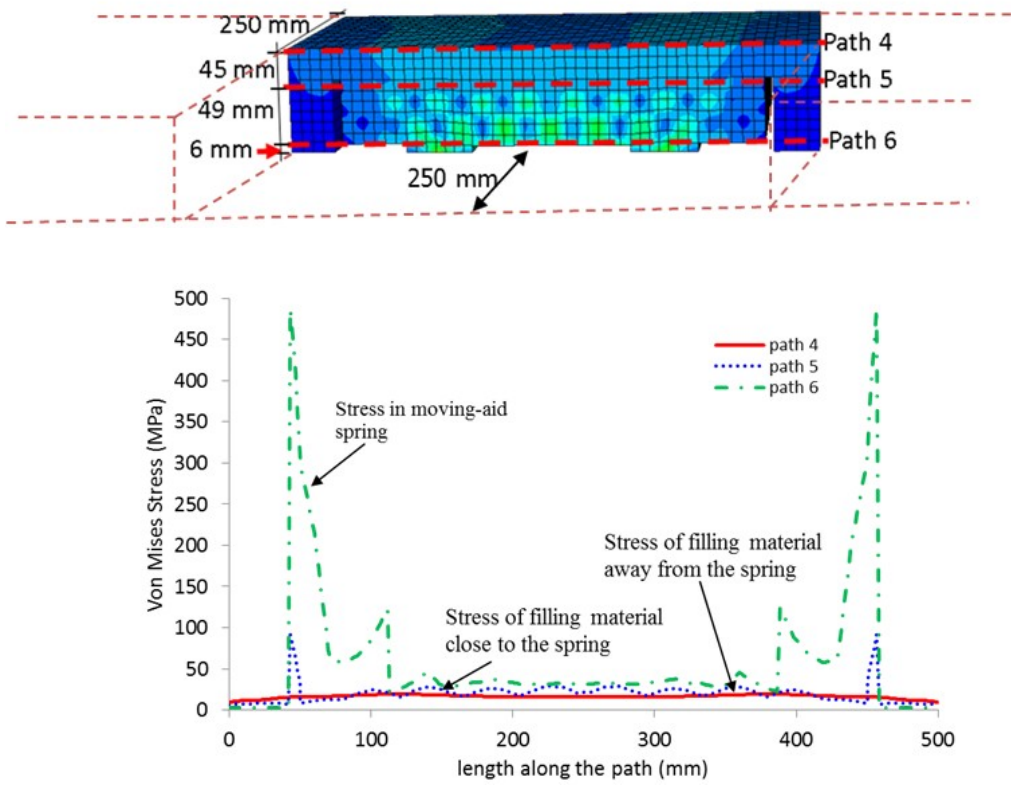

Figure 17. Variation of von Mises stress distribution along paths at different height of the model with movement-aid spring subjected to $+20 \mathrm{~mm}$ joint expansion at $-20^{\circ} \mathrm{C}$.

\section{Conclusions}

1. Experiments as well as FEM showed that the maximum strain of APJ is less than 0.1. This indicates that brittle type of cracks could occur at cold temperature $\left(-20^{\circ} \mathrm{C}\right)$ when the APJ is under tension from thermal contraction of the concrete structure. Furthermore, the lateral deformation with necking effect at the center of the APJ (X-Y plane) is larger than the vertical deformation along the center of the surface (X-Z plane). Moreover, the FEM parametric study of the joint length indicated that the necking effect has considerable influence up to $1 \mathrm{~m}$ length. This implies that attention should be paid and the necking effect should not be underestimated when working with APJ models of less than $1 \mathrm{~m}$ width. The vertical surface deformation (along X-Z plane) is not influenced by an increase in length. Note that the calibrated prototype APJ- $1^{\mathrm{a}}$ with $500 \mathrm{~mm}$ length captures the deformation trends reasonably well. Hence, a specimen with length above $500 \mathrm{~mm}$ can be used to predict the APJs performance under thermal load in reality.

2. The stress at critical points at the edge of the anti-deboning mat (point-C) and at the middle of the interface between cover plate and the APJ showed comparable stress values, irrespective of the APJ thickness of $60 \mathrm{~mm}$ or $100 \mathrm{~mm}$. Moreover, by changing the width of APJ, the stresses at these two specific points are not influenced as compared to the stresses at the interface between mastic asphalt and APJ. The significant change in stress at the interface between mastic asphalt and APJ (point A and B) can be attributed to the high strain and elongation of the APJs in case of narrow joint width. 
3. The use of anti-bonding mat helps distributing stresses more uniformly in the APJ system and can significantly minimize the stress concentration at the steel cover plate under tensile thermal loading. Further, from simulating contraction of the APJ follows, that the stress is reduced when using anti-bonding mat. This suggests that the bulging effect during contraction can be reduced, thus helping to maintain the smooth surface.

4. Using movement-aid springs helps in reducing the stress at critical points where crack propagation could occur. Introducing long anti-deboning mats could be helpful when using movement-aid springs. Since the filling material between the two L-profiles is not needed for transferring the stress to the concrete, the stress in the APJ will be fully transferred by the springs between the L-profile. In fact, movement-aids are practical means to distribute strain more uniformly and increase the movement capacity of APJ system.

\section{References}

ASTRA Guideline (2005), Fahrbahnubergange aus Polymerbitumen, Bundesamt fur Strassen. BBL Nr. 806.315.d. 2005.

Di Benedetto, H., Partl, M. N., Francken, L. \& Roche Saint André, C. (2001), Stiffness Testing for Bituminous Mixtures, Materials and Structures, 34(2), 66-70.

Bing, P., Kemao, Q., Huimin, X. \& Anand, A. (2009), Two-Dimensional Digital Image Correlation for inPlane Displacement and Strain Measurement: A Review, Measurement Science and Technology, 20(6), 062001.

Bramel, B. K., Dolan, C. W., Puckett, J. A. \& Ksaibati, K. (1999), Asphalt Plug Joints: Characterization and Specifications, University of Wyoming, Laramie.

Chu, T. C., Ranson, W. F. \& Sutton, M. A. (1985), Applications of Digital-Image-Correlation Techniques to Experimental Mechanics, Experimental Mechanics, 25(3), 232-244.

European Organisation for Technical Approvals. (2013), Guideline for European Technical Approval of Expansion Joints for Road Bridges, Kunstlaan 40 Avenue des Arts B - 1040 BRUSSELS.

Harvey J. A. F. \& Cebon D. (2003), Failure Mechanisms in Viscoelastic Films, Journal of Materials Science, 38(5), 1021-1032.

Hean, S. \& Partl, M. N. (2005), Performance O Two New Asphaltic Plug Joint Systems for Large Movements, TRB 2005 Annual Meeting

Sokolov, K., Gubler, R. \& Partl, M.N. (2005), Extended Numerical Modeling and Application of the Coaxial Shear Test for Asphalt Pavements Materials and Structures, 38, 515-522

Park, P., El-Tawil, S., Park, S. \& Naaman, A. (2010), Behavior of Bridge Asphalt Plug Joints under Thermal and Traffic Loads, Journal of Bridge Engineering, 15(3), 250-259.

Partl M. N, Hean S \& Poulikakos L. (2002), Asphaltic Plug Joint Characterization and Performance Evaluation, 9th international conference on asphalt pavements.

Partl, M. N. \& Hean, S. (2011), Experience with Testing and Performance Evaluation of Bituminous Plug Expansion Joint on Concrete Road Bridge, International Journal of Roads and Airports, 1:1-17.

Taylor, R. L., Pister, K. S. \& Goudreau, G. L. (1970), Thermomechanical Analysis of Viscoelastic Solids, International Journal for Numerical Methods in Engineering, 2(1), 45-59. 